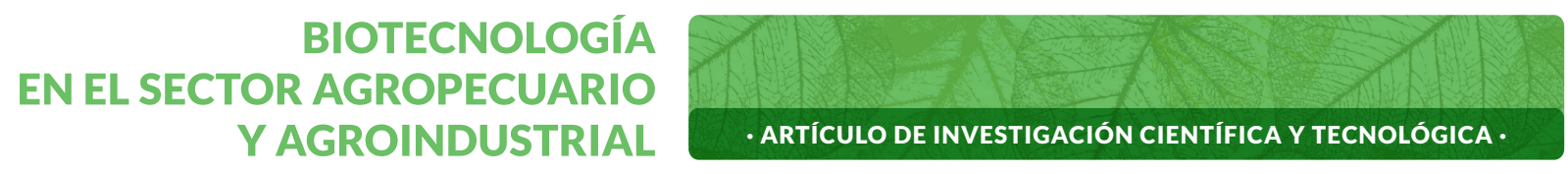

Vol. 19 No 2 · Julio - Diciembre 2021 • ISSN - 1692-3561 · ISSN-e 1909-9959 · https://doi.org/10.18684/bsaa.v19.n2.2021.1863

\title{
Desempeño de larvas y juveniles de Piaractus orinoquensis cultivados a diferentes densidades en sistemas con tecnología biofloc TBF
}

\section{Larvae and juveniles performance of Piaractus orinoquensis grown at different densities in systems with biofloc technology BFT}

\author{
COLLAZOS-LASSO, LUIS-FELIPE ${ }^{1}$; UENO-FUKURA, MITSUAKI'; \\ ARIAS-CASTELLANOS, JOSÉ-ALFREDO ${ }^{3}$; VINATEA-ARANA, LUIS-ALEJANDRO ${ }^{4}$
}

Historial del Artículo

Recibido para evaluación: 19 de Febrero 2021.

Aprobado para publicación: 22 de Abril 2021.

* Título del proyecto de origen: "Larvicultura y alevinaje de cachama blanca Piaractus orinoquensis, en sistema superintensivo de cultivo con tecnología biofloc - TBF". Financiación: Dirección general de investigaciones Universidad de los Llanos. Culminación: junio 2020.

1 Universidad de los Llanos, Instituto de Acuicultura de los Llanos IALL, Facultad de Ciencias Agropecuarias y recursos naturales, Grupos de Investigación IALL. Doctor en ciencias agrarias. Villavicencio, Colombia. https://orcid.org/0000-0002-6034-0037

2 Universidad de los Llanos, Instituto de Acuicultura de los Llanos IALL, Facultad de Ciencias Agropecuarias y recursos naturales, Grupos de Investigación IALL. Especialista en acuicultura. Villavicencio, Colombia. https://orcid.org/0000-0003-0209-975

3 Universidad de los Llanos, Instituto de Acuicultura de los Llanos IALL, Facultad de Ciencias Agropecuarias y recursos naturales, Grupos de Investigación IALL. PhD. en Ciencias biología. Villavicencio, Colombia. https://orcid.org/0000-0002-0980-5919

4 Universidade Federal de Santa Catarina, Departamento de Aquicultura, Laboratório de Camarões Marinhos. PhD. en Ciencias humanas. Florianópolis, Santa Catarina, Brazil. https://orcid.org/0000-0002-3893-4728 


\section{RESUMEN}

El sistema de cultivo de peces con TBF presenta condiciones de calidad de agua y oferta de alimento vivo favorables para el cultivo de peces con desarroIlo indirecto en etapas tempranas, en este sentido, el objetivo de este estudio fue evaluar el cultivo con TBF de larvas y juveniles de Piaractus orinoquensis a diferentes densidades de siembra. Para este fin se estableció biofloc a una relación carbono / nitrógeno de 15/1, evaluándose seis densidades de siembra, asi: $T 1=10, T 2=20$ y T3 $=30$ larvas $L^{-1}$ (peso inicial $=0,74 \pm 0,05 \mathrm{mg}$ y longitud inicial $=5,07 \pm 0,7 \mathrm{~mm}$ ) y T4 $=40, T 5=50$ y T6 $=60$ larvas $L^{-1}$ (peso inicial $=0,73 \pm 0,07 \mathrm{mg}$ y longitud inicial $=5,35 \pm 0,3 \mathrm{~mm}$ ). Los estudios se condujeron en dos fases: la primera de larvicultura, durante 12 días de cultivo, los resultados muestran que la supervivencia (\%) y el peso final fueron mayores en los tratamientos de menores densidades, sin embargo, los tratamientos con mayores densidades presentaron mayor número de larvas vivas. Para la segunda fase, comprendida entre el día 13 al 24 post siembra (DPS), se presentaron respuestas similares, siendo la supervivencia (\%) mayor a menores densidades de siembra (T1 = 79,8 $\pm 6,90 \%)$, no obstante, el número total de juveniles vivos fue mayor a densidades mayores (T6 $=34,5 \pm 5,2$ juveniles $\left.L^{-1}\right)$; el peso final disminuyó a medida que aumentó la densidad sin que la longitud presentara diferencias estadísticas. El análisis multivariado que relacionó la supervivencia, el peso, la longitud, el número de larvas y juveniles vivos, indicó que las mejores respuestas se presentaron en el T3 con una supervivencia del $49,5 \pm 7,4 \%$ y un número final de juveniles de 14,9 $\pm 2,1 \mathrm{~L}^{-1}$. La posibilidad de cultivar larvas y juveniles de P. orinoquensis de manera superintensiva con tecnología biofloc es factible en las condiciones reportadas, obteniendo los mejores resultados a una densidad de 30 larvas $L^{-1}$.

\section{ABSTRACT}

The fish culture system with BFT presents favorable conditions of water quality and supply of live food for the culture of fish with indirect development in early stages, in this sense, the present study aimed to evaluate the larvae and juveniles culture of Piaractus orinoquensis with BFT at different stocking densities. For this purpose, biofloc was established at a carbon/nitrogen ratio of 15/1, evaluating six stocking densities, as follows: $T 1=10, T 2=20, T 3=30$ larvae $L^{-1}$ (initial weight $=0,74 \pm 0,05 \mathrm{mg}$ and an initial length $=5,07 \pm 0,7 \mathrm{~mm}$ ) and $\mathrm{T} 4=40$, $T 5=50$ and $T 6=60$ larvae $L^{-1}$ (initial weight $=0,73 \pm 0,07 \mathrm{mg}$ and an initial length $=5,35 \pm 0,3 \mathrm{~mm}$ ). The studies were conducted in two phases: the first one of larviculture, during 12 days of culture, the results show that the survival (\%) and the final weight were higher in the treatments of lower densities, however,

Como citar este artículo: COLLAZOS-LASSO, LUIS-FELIPE; UENO-FUKURA, MITSUAKI; ARIAS-CASTELLANOS, JOSÉ-ALFREDO; VINATEA-ARANA, LUISALEJANDRO. Desempeño de larvas y juveniles de Piaractus orinoquensis cultivados a diferentes densidades en sistemas con tecnología biofloc TBF. Biotecnología en el sector agropecuario y agroindustrial, v. 19, n. 2, 2021, p. 201-213. Doi: https://doi. org/10.18684/bsaa.v19.n2.2021.1864

\section{PALABRAS CLAVE:}

Piaractus orinoquensis; Biofloc; Densidad; Larva; Juvenil; Supervivencia; Peso; Longitud; Relación carbono/nitrógeno;

Sistema de cultivo.

\section{KEY WORDS:}

Piaractus orinoquensis; Biofloc; Density; Larvae; Juvenile; Survival; Weight; Length; Carbon/nitrogen ratio; Culture system. 
the treatments with higher densities presented greater number of live larvae. For the second phase, from day 13 to 24 post-stocking (DPS), similar responses to larviculture were presented, being that survival (\%) was higher at lower stocking densities (T1 = 79,8 \pm 6,90\%), however, the total number of live juveniles was higher at higher densities (T6 = 34,5 $\pm 5,2$ juveniles $L^{-1}$ ); the final weight decreased as the density increased without the length presenting statistical differences. The multivariate analysis that related survival, weight, length, number of live larvae and juveniles, indicated that the best responses were presented in T3 with a survival of $49,5 \pm 7,4 \%$ and a final number of juveniles of 14,9 $\pm 2,1 \mathrm{~L}^{-1}$. The possibility of cultivating larvae and juveniles of $P$. orinoquensis in a superintensive way with biofloc technology is feasible under the reported conditions, obtaining the best results at a density of 30 larvae $L^{-1}$.

\section{INTRODUCCIÓN}

Las cifras de la piscicultura en Colombia, muestran al género Piaractus, con las especies P. brachypomus y P. orinoquensis, Ilamadas cachama blanca, en el segundo renglón de la producción nacional, con una participación para el año 2019 del $19 \%$ de las 165.444 toneladas reportadas por el Sistema de Información de Gestión y Desempeño de Organización de Cadenas del Ministerio de agricultura y desarrollo rural de Colombia (SIOC, 2020). Para suplir esta demanda, los productores de semilla de cachama blanca han establecido técnicas de reproducción inducida con hormonas, de incubación y posterior a la reabsorción del vitelo (90-94 horas poseclosión HPE) de cultivo de larvas; esta última, se práctica en estanques en tierra previamente abonados, hasta cosechar lo que se conoce entre productores como alevinos (Atencio, 2001), el número producido es irregular, teniendo en cuenta que estos sistemas están expuestos a factores externos adversos, como el clima y la presencia de depredadores (Prieto y Atencio, 2008).

Teniendo en cuenta lo anterior, la implementación de nuevas tecnologías en la producción piscícola es una necesidad, que permita tener un mayor control del sistema, incrementando el rendimiento del cultivo y generando menores impactos, un ejemplo de esto es la tecnología biofloc, la cual busca tratar los residuos propios de la acuicultura, removiendo los compuestos nitrogenados producto de las adiciones de alimento y el metabolismo de las especies cultivadas (Browdy et al., 2012), evitando de esta manera la muerte por toxicidad, en especial del amoniaco $\left(\mathrm{NH}_{3}\right)$ y el nitrito $\left(\mathrm{NO}_{2}{ }^{-}\right)$para peces dulceacuícolas (Baldisserotto, 2013); esta condición se logra a partir de la asimilación y oxidación del N, por microorganismos aerobios con actividades autotróficas y heterotróficas a una alta relación C/N (Crab et al., 2007; Azim et al., 2008; De Shryver et al., 2008; Kubitza, 2011) resolviendo de esta manera los problemas de exceso de nutrientes en la producción a partir de su reciclaje, generando menores impactos, gastos de agua y descargas al medio ambiente (Avnimelech, 2015).

La dominancia de microorganismos heterótrofos y autótrofos que se promueven en los sistemas biofloc para la eliminación biológica del nitrógeno, depende de las relaciones carbono/nitrógeno (Gomes Vilani et al., 2016; Maya-Gutiérrez et al., 2016; Luo et al., 2017), sugiriendo que una mayor relación promueve vías heterótrofas, afirmación que es corroborada en el trabajo de Bakar et al. (2015) cuando presentan la reducción del N a relaciones $\mathrm{C} / \mathrm{N}$ de 10, 15, 20, 25 y 30/1, siendo que a una mayor proporción de $\mathrm{C} / \mathrm{N}$ menores fueron los procesos de nitrificación; estas relaciones $\mathrm{C} / \mathrm{N}$ presentan diferencias en la conformación orgánica del biofloc, estando relacionadas proporcionalmente con un mayor aporte de carbono orgánico al sistema (Martins et al., 2017) de esta manera las vías heterótrofas tienen como producto una mayor producción de sólidos en suspensión volátiles (SSV) (Ebelin et al., 2006; Xu et al., 2016), generando condiciones particulares en la conformación de los eslabones superiores de la red trófica (Ray et al., 2010; Hernández et al., 2017; Ayazo-Genes et al., 2019).

Los estudios de estos consorcios bacterianos a los que se les ha llamado bioflocs, se han desarrollado con gran expectativa en el cultivo de camarones y peces en etapas de levante y engorde (Hargreaves, 2013; Avnimelech, 2015). En los últimos años se han realizado investigaciones de la implementación de la TBF en el cultivo de larvas y juveniles de peces dulciacuícolas, ejemplo de ello son los reportes para tilapia nilótica Oreochoromis niloticus (Ekasari et al., 2015; García-Ríos et al., 2019); bagre africano Clarias gariepinus (Fauji et al., 2018) y el bagre sudamericano Ramdia quelen (Poli et al., 2015); otros estudios, reportan el cultivo con TBF de peces en etapas tempranas a 
diferentes densidades (Liu et al.,2018; Fauji et al., 2018 y Diatin et al., 2019) permitiendo esclarecer la capacidad de carga del sistema, lo cual está relacionado con las condiciones del biofloc y la ontogenia de la especie de cultivo.

Sin embargo, no se presentan reportes del cultivo en biofloc de peces con ontogenia indirecta en etapas tempranas, siendo que este íctioplancton con características altriciales lecitotróficas (Balón, 1984) requiere estrictamente alimento vivo durante el inicio de la exotrofia. En este sentido, el objetivo de este trabajo fue evaluar el desempeño de larvas y juveniles de Piaractus orinoquensis cultivados en sistemas con bioflocs a diferentes densidades.

\section{MÉTODO}

Los experimentos se realizaron en el Instituto de Acuicultura de los Llanos (IALL), adscrito a la Facultad de Ciencia Agropecuarias y Recursos Naturales de la Universidad de los Llanos, Villavicencio - Colombia $\left(4^{\circ} 04^{\prime} 30^{\prime \prime} \mathrm{N}\right.$ $\left.73^{\circ} 35^{\prime} 07^{\prime \prime} \mathrm{O}\right)$, a una altitud de 418 m.s.n.m., temperatura ambiental media de $27^{\circ} \mathrm{C}$, humedad relativa del $75 \%$ y precipitación pluvial de $4050 \mathrm{~mm} / \mathrm{año}$.

\section{Material biológico}

Las larvas de $P$. orinoquensis se obtuvieron a partir de reproducciones inducidas con extracto pituitario de carpa. La incubación, eclosión y reabsorción de saco vitelino, se realizó en incubadoras cónicas de flujo ascendente con volumen de 200 L, conectadas a un sistema de recirculación en acuicultura (RAS) hasta las 92 horas post eclosión (hpe).

\section{Establecimiento del biofloc (macrocosmos)}

Para el establecimiento del biofloc se utilizó un tanque circular de $3 \mathrm{~m}$ de diámetro revestido con una bolsa de geomembrana (volumen útil de $7000 \mathrm{~L}$ ), cubierto por un domo plástico con $90 \%$ de transparencia. El sistema de aireación lo conformó un soplador de $1 \mathrm{HP}$ conectado a un sistema difusor constituido de $12 \mathrm{~m}$ de manguera microperforada Aero-Tube ${ }^{\mathrm{TM}}$ distribuida en tres anillos en el fondo. Se llenó el tanque con $7000 \mathrm{~L}$ de agua y se manejó una relación C/N de 15/1, teniendo en cuenta los planteamientos descritos por De Shyver et al. (2008) y Avnimelech (2015). Como fuente de nitrógeno se utilizó alimento balanceado con $32 \%$ de proteína (humedad del $13 \%$ ), como fuente de carbono orgánico (CO) fue adicionada melaza de caña de azúcar (33,65\% de CO total) y se adicionó bicarbonato de sodio $\left(\mathrm{NaHCO}_{3}\right)$ para regular la alcalinidad. Las adiciones de alimento balanceado fueron diarias y se calculó la cantidad necesaria para incrementar teóricamente en $2 \mathrm{mg} \mathrm{L}^{-1}$ el nitrógeno amoniacal total NAT $\left(\mathrm{NH}_{4}{ }^{+}+\mathrm{NH}_{3} \mathrm{mg} \mathrm{L}^{-1}\right)$, según los lineamientos de Fauji et al. (2018), hasta que las concentraciones de NAT y nitrito $\left(\mathrm{NO}_{2}^{-}\right.$) estuvieran en rangos seguros para peces (Jiménez et al., 2018).

\section{Diseño experimental}

Se evaluó el desempeño productivo de larvas y juveniles de $P$. orinoquensis a seis densidades de siembra, así: $T 1=$ $10, \mathrm{~T} 2=20, \mathrm{~T} 3=30, \mathrm{~T} 4=40, \mathrm{~T} 5=50$ y T6 $=60$ larvas $\mathrm{L}^{-1}$. Cada tratamiento con cuatro réplicas, las cuales consistieron en jaulas flotantes circulares con volumen útil de $7,8 \mathrm{~L}$, en material plástico recubiertas en un $80 \%$ de la estructura con malla SEFAR ${ }^{\circledR}$ de $650 \mu$ m de apertura del ojo de malla, las cuales se sumergieron en el macrocosmos. Las densidades fueron evaluadas en dos fases: de larvicultura y juveniles, como se presenta a continuación:

\section{Larvicultura de P. orinoquensis cultivadas a diferentes densidades en biofloc}

Las densidades se evaluaron en dos grupos, en dos tiempos diferentes. El primer grupo de tratamientos fueron los T1:10, T2:20 y T3:30 larvas $\mathrm{L}^{-1}$ de $92 \mathrm{hpe}$, las larvas fueron sembradas en cada réplica con un peso inicial de $0,74 \pm 0,05 \mathrm{mg}$ y longitud inicial de 5,07 $\pm 0,7 \mathrm{~mm}$. Para el segundo grupo de tratamientos T4:40, T5: 50 y T6: 60 larvas $\mathrm{L}^{-1}$ de $92 \mathrm{hpe}$, el peso inicial fue de 0,73 $\pm 0,07 \mathrm{mg}$ y la longitud inicial de $5,35 \pm 0,3 \mathrm{~mm}$, el tiempo de cultivo 
para ambos grupos fue de 12 días (del día 0 al 12 DPS). Previo a la siembra de las larvas para cada tratamiento y réplica, se realizó durante 2 horas una aclimatación con agua de biofloc.

\section{Cultivo de juveniles de $P$. orinoquensis cultivados a diferentes densidades en biofloc}

Esta etapa fue continua a los experimentos de larvicultura, del día 13 al 24 DPS y también fue evaluada en dos grupos en dos tiempos diferentes, así: el primer grupo se evaluaron los tratamientos T1: 10, T2: 20 y T3: 30 larvas $\mathrm{L}^{-1}$, con peso inicial de $8,1 \pm 1,9 \mathrm{mg}$ y longitud inicial de $7,5 \pm 1,2 \mathrm{~mm}$, en el segundo grupo se evaluaron los tratamientos T4: 40, T5: 50 y T6:60 larvas $\mathrm{L}^{-1}$, cuyo peso promedio inicial fue de 7,9 $\pm 2,2 \mathrm{mg}$, con una longitud total inicial de $7,8 \pm 1,6 \mathrm{~mm}$.

Durante las dos fases evaluadas (larvicultura y alevinaje), los macrocosmos fueron sembradas con 30 larvas L-1 con el objetivo de garantizar condiciones equilibradas entre las réplicas y el exterior.

\section{Calidad del agua}

Durante los ensayos se realizaron mediciones y registros de la calidad del agua del macrocosmos para los dos grupos de tratamientos evaluados bajo las siguientes consideraciones: una vez al día (am), se midieron las concentraciones de nitrógeno amoniacal total NAT, nitrito $\left(\mathrm{NO}_{2}^{-} \mathrm{mg} \mathrm{L}^{-1}\right)$, nitrato $\left(\mathrm{NO}_{3}^{-} \mathrm{mg} \mathrm{L}^{-1}\right)$ y alcalinidad total (AT mg CaCO $\mathrm{L}^{-1}$ ) con fotómetro YSI Yellow Spring Instrument ${ }^{\text {TM }}$ Ref 9500 Exactitud $\pm 0,5 \%$ a $4 \%$ transmitancia \pm 0,005 a 0,3 AU Resolución 0,001 AU, y sólidos sedimentables (SS $\mathrm{ml} \mathrm{L}^{-1}$ ) con conos Imhoff conforme a la metodología de APHA (1998), adaptada por Avnimelech (2007). Dos veces al día fueron registrados la temperatura (T $\left.{ }^{\circ} \mathrm{C}\right)$, el pH y el oxígeno $\left(\mathrm{O}_{2} \mathrm{mg} \mathrm{L}^{-1} \mathrm{y} \%\right)$ con sonda multiparamétrica HANNA HI98196.

Antes de la siembra de larvas y juveniles (día cero), se tomaron muestras del agua y por triplicado fueron procesadas para sólidos totales (ST), sólidos volátiles (SV) y sólidos fijos (SF), mediante método gravimétrico siguiendo la metodología da APHA (2017 - 2540B y 2017- 2540E, respectivamente).

\section{Desempeño productivo de larvas y juveniles}

En cuanto a la productividad de los peces, se realizaron los conteos para supervivencia $(\mathrm{S})$ en \% $=(\mathrm{Nf} / \mathrm{Ni}) \times 100$ donde, Nf: número de individuos al final del experimento y Ni: número de individuos al inicio del experimento. Las mediciones (longitud, $\mathrm{mm}$ ) y pesajes $(\mathrm{mg})$ de las larvas y juveniles se realizaron al inicio y al final de cada experimento, tomando una muestra de larvas de cada unidad experimental correspondiente al $20 \%$ del total. La medición y conteo de las larvas y juveniles se realizó mediante el programa ImageJ versión 1.52p (https:// imagej.nih.gov/ij/.) con java 8.0.2010.9 (Bourne, 2010; Andrade-Vera et al., 2017) a partir del procesamiento de fotografías tomadas con cámara Nikon D3500 Digital SLR with 18-55mm Lens. Para el pesaje, balanza analítica (Mettler Toledo AB 204-S sensibilidad de 0,0001, Columbus, Ohio-43240 USA).

\section{Análisis estadístico}

Se empleó el modelo lineal general mediante diseño de estructura experimental de una vía de efecto fijo desbalanceado, incorporando la técnica Manova, determinando la dimensionalidad por medio de la función de máxima verosimilitud, empleando transformación de datos por medio de la técnica arco seno para las variables expresadas en porcentaje (supervivencia, saturación de oxígeno). Las comparaciones unidimensionales se realizaron por medio de la prueba de Tukey, teniendo en cuenta un nivel de significancia del $5 \%(p<0,05)$, usando letras diferentes para indicar diferencias estadísticas significativas. Se utilizó el paquete estadístico SAS University. 


\section{RESULTADOS}

\section{Cultivo de larvas de $P$. orinoquensis en bioflocs a diferentes densidades}

Calidad de agua. Como se observa en el cuadro 1, se presentaron diferencias estadísticas para todos los parámetros entre grupos de tratamientos, excepto para NAT, AT y SS.

Cuadro 1. Variables físicas y químicas del agua en el cultivo de larvas de $P$. orinoquensis con tecnología biofloc a diferentes densidades $\left(10,20,30\right.$ y 40, 50, 60 larvas $\left.\mathrm{L}^{-1}\right)$ desde el día 0 hasta el 12 DPS.

\begin{tabular}{|l|c|c|}
\hline \multicolumn{1}{|c|}{ Parámetros } & T1, T2 y T3 & T4, T5 y T6 \\
\hline Temperatura $\left({ }^{\circ} \mathrm{C}\right)$ & $28,1 \pm 0,6^{\mathrm{a}}$ & $27,4 \pm 0,6^{\mathrm{b}}$ \\
\hline Oxígeno disuelto $\left(\mathrm{mg} \mathrm{L}^{-1}\right)$ & $6,5 \pm 0,4^{\mathrm{b}}$ & $6,8 \pm 0,5^{\mathrm{a}}$ \\
\hline Saturación de oxígeno (\%) & $88,3 \pm 4,4^{\mathrm{b}}$ & $91,4 \pm 6,0^{\mathrm{a}}$ \\
\hline $\mathrm{pH}$ & $7,5-8,2$ & $7,9-8,4$ \\
\hline Nitrógeno amoniacal total $\left(\mathrm{mg} \mathrm{L}^{-1}\right)$ & $0,4 \pm 0,1$ & $0,26 \pm 0,22$ \\
\hline Nitrito $\mathrm{NO}_{2}^{-}\left(\mathrm{mg} \mathrm{L}^{-1}\right)$ & $1,0 \pm 0,5^{\mathrm{a}}$ & $0,2 \pm 0,1^{\mathrm{b}}$ \\
\hline Nitrato $\mathrm{NO}_{3}^{-}\left(\mathrm{mg} \mathrm{L}^{-1}\right)$ & $292,4 \pm 74,4^{\mathrm{a}}$ & $178,7 \pm 34,6^{\mathrm{b}}$ \\
\hline Alcalinidad total $\left(\mathrm{mg} \mathrm{CaCO}_{3} \mathrm{~L}^{-1}\right)$ & $177,3 \pm 28,8$ & $187,0 \pm 22,6$ \\
\hline Sólidos sedimentables $\left(\mathrm{ml} \mathrm{L}^{-1}\right)$ & $43,0 \pm 2,2$ & $41,0 \pm 4,6$ \\
\hline
\end{tabular}

Datos presentados como media \pm desviación estándar.

Letras distintas indican diferencia significativa $(p<0,05)$

Las concentraciones teóricas de amonio no ionizado $\left(\mathrm{NH}_{3}\right)$ presentes en el NAT (Emerson et al., 1975) de los dos grupos de tratamientos ( $\mathrm{G} 1: 0,021 \pm 0,005$ y G2: $0,064 \pm 0,16 \mathrm{mg} \mathrm{L}^{-1}$ ), fueron inferiores a las $\mathrm{CL} 50$ reportadas para larvas de Oreochromis niloticus (1,007-1,01 $\mathrm{mg} \mathrm{L}^{-1} \mathrm{de} \mathrm{NH}_{3}$ ) expuestas durante 48 horas a un pH 8,0 $\pm 0,2$ y $\mathrm{T}=23^{\circ} \mathrm{C}$ (Karasu y Koksal, 2005). En cuanto al nitrito $\left(\mathrm{NO}_{2}^{-}\right)$, la segunda principal forma tóxica de nitrógeno en piscicultura (Baldisserotto, 2013) las concentraciones fueron inferiores a las reportada por Ekasari et al. (2015) y García-Ríos et al. (2019) para larvas de tilapia cultivadas en biofloc $\left(2,04 \pm 0,35 \mathrm{mg} \mathrm{L}^{-1} \mathrm{de} \mathrm{NO}_{2}\right.$ - a una relación $\mathrm{C} / \mathrm{N}$ de $\left.10 / 1\right)$. En este sentido, las concentraciones de las diferentes formas del nitrógeno en los dos grupos experimentales fueron menores a los niveles propuestos para el cultivo de peces en biofloc por Emerenciano et al. (2017).

En cuanto al $\mathrm{pH}$ y la alcalinidad, en todos los tratamientos presentaron valores similares a los reportados para el cultivo de larvas de Rhamdia quelen en biofloc (Poli et al., 2015), indicando el adecuado efecto buffer en las aguas experimentales (Kubitza, 2017). Las concentraciones y saturaciones de oxígeno, así como la temperatura, se registraron en rangos propuestos para el cultivo de peces en biofloc por Emerenciano et al. (2017).

Los SS (cuadro 1), ST, SV y SF (cuadro 2) no presentaron diferencias significativas entre los dos grupos de tratamientos, hecho que parece indicar condiciones similares de los sólidos durante el cultivo de larvas. Los rangos de SS y SV fueron similares a los propuestos por Emerenciano et al. (2017) y reportados por Pérez-Fuentes et al. (2016) y Zapata et al. (2017) para el cultivo de larvas de tilapia. Los SV estuvieron en rangos similares a los reportados en biofloc por Azim et al. (2008), con valores cercanos a los $300 \mathrm{mg}$ L-1 de SSV. Para el cultivo de larvas de Rhamdia quelen, Poli et al. (2015) encontraron supervivencias sin diferencia significativa en rangos de concentración de SST entre 200 y 1000 mg L-1. De este modo, los valores reportados en este estudio sugieren una alta tolerancia de las larvas de cachama a altas concentraciones de sólidos en el cultivo con TBF. 
Cuadro 2. Análisis comparativo de sólidos del agua en el cultivo de larvas de P. orinoquensis en sistema con tecnología biofloc a diferentes densidades.

\begin{tabular}{|l|l|l|}
\hline & \multicolumn{1}{|c|}{ T1, T2 y T3 } & \multicolumn{1}{c|}{ T4, T5 y T6 } \\
\hline Sólidos totales $\left(\mathrm{ST} \mathrm{mg} \mathrm{L}^{-1}\right)$ & $2367,8 \pm 21,6$ & $2015,5 \pm 920$ \\
\hline Sólidos volátiles $\left(\mathrm{SV} \mathrm{mg} \mathrm{L}^{-1}\right)$ & $392,2 \pm 21,6$ & $395,5 \pm 75$ \\
\hline Sólidos fijos $\left(\mathrm{SF} \mathrm{mg} \mathrm{L}^{-1}\right)$ & $1975,5 \pm 2$ & $1620,0 \pm 870$ \\
\hline
\end{tabular}

Datos presentados como media \pm desviación estándar. Letras distintas indican diferencia significativa $(p<0,05)$.

Parámetros productivos. Los mayores porcentajes de supervivencia se presentaron en T1 y T2, con diferencia significativa $(p<0,05)$ respecto a T3, T4, T5 y T6, sin embargo, el número total de larvas vivas fue mayor en los T5 y T6 con diferencia respecto a los otros tratamientos (Cuadro 3 y Figura 1). Estos resultados de desempeño productivo de larvas de P. orinoquensis, indican que a mayor densidad de siembra la supervivencia fue menor, consideración similar a la reportada por Liu et al. (2018), Fauji et al. (2018) y Diatin et al. (2019). Esta relación inversa entre la densidad de siembra y la supervivencia sugiere que la capacidad de carga del sistema además del mantenimiento de las condiciones de calidad de agua en rangos de confort, se ve limitada por la biodisponibilidad de alimento vivo (plancton en general), condición y requerimiento fundamental durante la fase larval de cachama blanca, especie altricial con una ontogenia indirecta (Atencio, 2001; David-Ruales et al., 2018). Cuando se evalúa el número de larvas vivas al final del experimento, los tratamientos con mayores densidades de siembra (T5 y T6), presentan mayor número larvas vivas en relación con los demás tratamientos, siendo el mayor el T6 con una supervivencia del 47,7 $\pm 3,2 \%$, respuestas similares a las reportadas por Diatin et al. (2019) para Corydoras aeneus $\left(50,42 \pm 3 \%\right.$ a densidad de 6000 peces $\left./ \mathrm{m}^{3}\right)$. En esta fase larvaria el comportamiento de la supervivencia versus el número final de larvas vivas fue similar a lo reportado por Fauji et al. (2018) para larvas de Clarias gariepinus, quienes encontraron que al aumentar la densidad de peces (de 4,6 , y 8 peces $\mathrm{L}^{-1}$ ), se obtiene una menor supervivencia, pero un mayor número de peces producidos.

Respuesta y tendencias similares se presentaron para el peso final, siendo que, en el T1 con menores densidades, el peso final fue mayor y con diferencias significativas $(p<0,05)$ respecto a los $T 2$ y T3, seguido de los T4 y T5, este último no presentó diferencia respecto al T6. En cuanto a la longitud final no se presentaron diferencias entre tratamientos.

Cuadro 3. Análisis biométricos de larvas de 12 DPS de P. orinoquensis cultivadas en sistemas con tecnología biofloc a diferentes densidades $\left(10,20,30\right.$ y $40,50,60$ larvas $\left.L^{-1}\right)$.

\begin{tabular}{|c|c|c|c|c|c|c|}
\hline & T1 & T2 & T3 & T4 & T5 & T6 \\
\hline $\operatorname{LTF}(\mathrm{mm})$ & $13,6 \pm 1,7$ & $13,3 \pm 1,5$ & $13,7 \pm 1,4$ & $10,7 \pm 1,7$ & $11,1 \pm 1,7$ & $11,3 \pm 1,7$ \\
\hline PMF (mg) & $41,8 \pm 13,1^{\mathrm{a}}$ & $37,7 \pm 11,3^{b}$ & $35,9 \pm 9,4^{b}$ & $13,9 \pm 6,5^{d}$ & $14,7 \pm 6,4^{\mathrm{dc}}$ & $17,6 \pm 6,4^{c}$ \\
\hline $\mathrm{S}(\%)$ & $83,9 \pm 11,8^{a}$ & $63,9 \pm 8,8^{a}$ & $55,8 \pm 8,0^{b}$ & $46,9 \pm 7,2^{b}$ & $51,8 \pm 5,8^{b}$ & $47,7 \pm 3,{ }^{2} b$ \\
\hline NTL $\left(N^{\circ}\right)$ & $8,3 \pm 1,2^{d}$ & $12,8 \pm 1,8^{c}$ & $16,7 \pm 2,4^{\mathrm{bc}}$ & $18,7 \pm 2,6^{b}$ & $25,9 \pm 2,9^{a}$ & $28,6 \pm 1,9^{a}$ \\
\hline Dimensionalidad & \multicolumn{6}{|c|}{ Determinación de la Dimensionalidad } \\
\hline Valor propio & \multicolumn{2}{|l|}{ Proporción } & $\mathrm{F}$ & $\operatorname{Pr}>\mathrm{F}$ & \multicolumn{2}{|l|}{ Significancia } \\
\hline 1 & \multicolumn{2}{|l|}{0,9144} & 2,0 & 0,0345 & \multicolumn{2}{|l|}{ Significativo } \\
\hline 2 & \multicolumn{2}{|l|}{0,0724} & 0,5 & 0,8851 & \multicolumn{2}{|l|}{ No Significativo } \\
\hline 3 & \multicolumn{2}{|l|}{0,0132} & 0,2 & 0,8967 & \multicolumn{2}{|l|}{ No Significativo } \\
\hline MANOVA & \multicolumn{6}{|c|}{ Análisis Multivariado de la Varianza } \\
\hline Prueba & \multicolumn{2}{|l|}{ VALOR } & $\mathrm{F}$ & $\mathrm{Pr}>\mathrm{F}$ & \multicolumn{2}{|l|}{ Significancia } \\
\hline Wilks' Lambda & \multicolumn{2}{|l|}{0,27388762} & 2,0 & 0,0345 & \multicolumn{2}{|l|}{ Significativo } \\
\hline Pillai's Trace & \multicolumn{2}{|l|}{0,84067872} & 1,6 & 0,1142 & \multicolumn{2}{|l|}{ No Significativo } \\
\hline Hotelling-Lawley T & \multicolumn{2}{|l|}{2,24271035} & 2,6 & 0,0142 & \multicolumn{2}{|l|}{ Significativo } \\
\hline \multirow[t]{3}{*}{ Roy's Greatest } & \multicolumn{2}{|l|}{2,05071261} & 8,2 & 0,0002 & \multicolumn{2}{|l|}{ Significativo } \\
\hline & \multicolumn{6}{|c|}{ Análisis Canónico } \\
\hline & T1 ${ }^{\text {a }}$ & $\mathrm{T} 2^{\mathrm{b}}$ & T3 ${ }^{b}$ & T4 ${ }^{d}$ & T5 ${ }^{d}$ & T6 ${ }^{c}$ \\
\hline
\end{tabular}

Datos presentados como media \pm desviación estándar, Letras distintas indican diferencia significativa $(p<0,05)$. LTF: longitud corporal total final. PMF: peso corporal medio final. S: supervivencia. NTL: número total de larvas vivas $\mathrm{L}^{-1}$ 

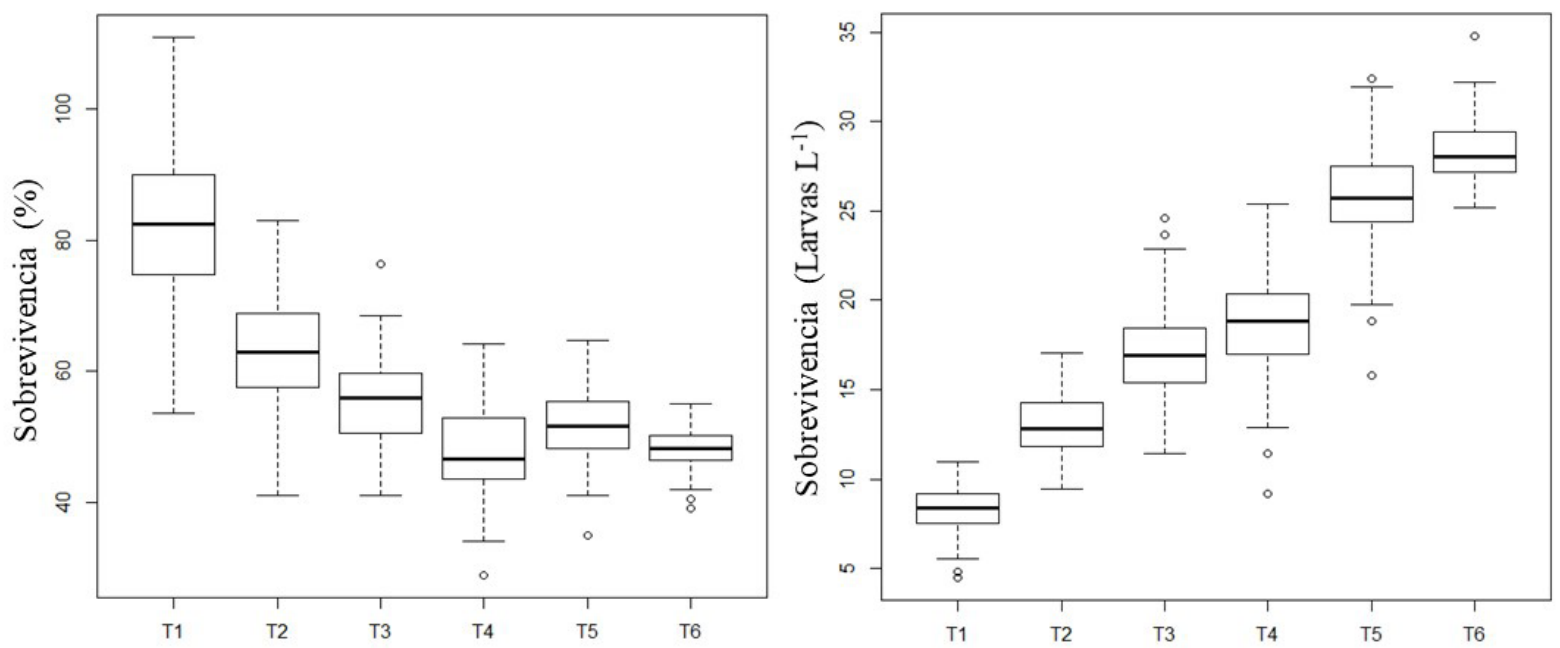

Figura 1. Sobrevivencia en porcentaje y número de larvas $\mathrm{L}^{-1}$ de $P$. orinoquensis cultivadas durante 12 días con tecnología biofloc a diferentes densidades (Kruskal-Wallis test with a p.value: 0,05).

\section{Cultivo de larvas hasta juveniles de $P$. orinoquensis en sistemas con tecnología biofloc a diferentes densidades}

Calidad de agua. Como se puede observar en el cuadro 4 , hubo diferencias significativas $(p<0,05)$ entre los dos grupos de tratamientos para las variables temperatura, oxígeno disuelto, nitrito y alcalinidad total.

Cuadro 4. Variables físicas y químicas del agua en el cultivo de larvas hasta juveniles de $P$. orinoquensis en sistema con tecnología biofloc desde el día 13 hasta el 24 DPS a diferentes densidades (10, 20, 30 y 40, 50, 60 juveniles L-1)

\begin{tabular}{|l|c|c|}
\hline \multicolumn{1}{|c|}{ Variables } & T1, T2 y T3 & T4, T5 y T6 \\
\hline Temperatura $\left({ }^{\circ} \mathrm{C}\right)$ & $28,2 \pm 0,6^{\mathrm{a}}$ & $26,7 \pm 0,5^{\mathrm{b}}$ \\
\hline Oxígeno disuelto $\left(\mathrm{mg} \mathrm{L}^{-1}\right)$ & $6,6 \pm 0,3^{\mathrm{b}}$ & $6,9 \pm 0,3^{\mathrm{a}}$ \\
\hline Saturación de oxígeno (\%) & $88,9 \pm 3,7$ & $90,3 \pm 3,2$ \\
\hline $\mathrm{pH}$ & $7,7-8,2$ & $7,5-8,1$ \\
\hline Nitrógeno amoniacal total $\left(\mathrm{mg} \mathrm{L}^{-1}\right)$ & $0,72 \pm 0,7$ & $1,08 \pm 1,16$ \\
\hline Nitrito $\mathrm{NO}_{2}^{-}\left(\mathrm{mg} \mathrm{L}^{-1}\right)$ & $0,61 \pm 0,5^{\mathrm{a}}$ & $0,25 \pm 0,3^{\mathrm{b}}$ \\
\hline Nitrato $\mathrm{NO}_{3}^{-}\left(\mathrm{mg} \mathrm{L}^{-1}\right)$ & $255,8 \pm 117,5$ & $217,7 \pm 39,8$ \\
\hline Alcalinidad total $\left(\mathrm{mg} \mathrm{CaCO}_{3} \mathrm{~L}^{-1}\right)$ & $235,7 \pm 23,7^{\mathrm{a}}$ & $171,2 \pm 11,4^{\mathrm{b}}$ \\
\hline Sólidos sedimentables $\left(\mathrm{ml} \mathrm{L}^{-1}\right)$ & $42,0 \pm 6,43$ & $46,6 \pm 7,5$ \\
\hline
\end{tabular}

Datos presentados como media \pm desviación estándar. Letras distintas indican diferencia significativa $(p<0,05)$

Durante esta fase comprendida entre el día 13 al 24 DPS, los parámetros de calidad de agua estuvieron en rangos similares a lo reportado para la larvicultura y apropiados para el cultivo de peces con tecnología biofloc en cuanto a temperatura, saturación y concentración de oxígeno, pH y alcalinidad total (Emerenciano et al., 2017).

En cuanto al NAT, los diferentes tratamientos resultaron con valores similares a los reportados en trabajos de larvicultura en biofloc de Rhamdia quelen (Poli et al., 2015); Clarias gariepinus (Ekasari et al., 2016) y Oreochromis niloticus (Li et al., 2018), e inferiores a la concentración letal (CL50) reportada para juveniles (1,2 g) de Piaractus mesopotamicus (Barbieri y Vigliar Bondioli, 2013), siendo esta última especie del mismo género de la cachama blanca. De igual manera, cuando se calculó la concentración de amonio reducido no ionizado $\left(\mathrm{NH}_{3}\right)$ presente en 
el NAT para los dos grupos de tratamientos a partir de los planteamientos de Emerson et al. (1975), estos fueron menores al CL50 de juveniles de P. mesopotamicus (Barbieri y Vigliar Bondioli, 2013) y de tilapia del Nilo (Karasu y Koksal, 2005). En los dos grupos de tratamientos el nitrito estuvo en rangos inferiores a los reportados en poslarvas de tilapia del Nilo (Ekasari et al., 2015) y Rhamdia quelen (Poli et al., 2015) cultivadas en biofloc. Los sólidos sedimentables se presentaron dentro de valores similares a los reportados para el cultivo de tilapia nilótica (Pérez-Fuentes et al., 2016; Zapata et al., 2017).

Desempeño productivo. El análisis biométrico mostró la continuidad del comportamiento de la larvicultura. Hubo diferencias significativas entre los dos grupos para el crecimiento en peso y el porcentaje de supervivencia, pero no para para longitud (Cuadro 5 y Figura 2).

Cuadro 5. Desempeño productivo de larvas hasta juveniles (13 - 24 DPS) de P. orinoquensis, cultivadas con tecnología biofloc a diferentes densidades.

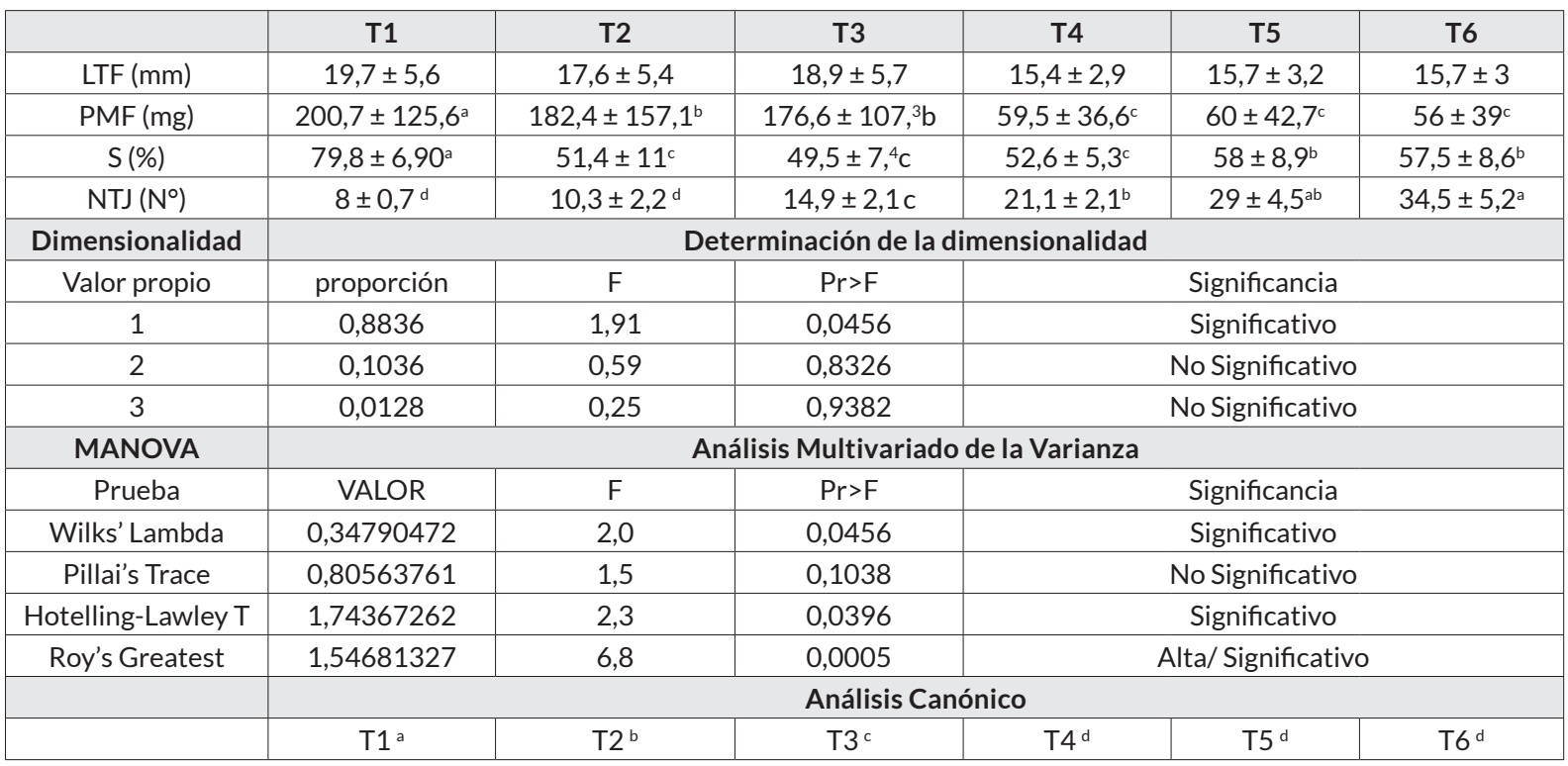

Datos presentados como media \pm desviación estándar. Letras distintas indican diferencia significativa $(p<0,05)$. LTF: longitud corporal total final. PMF: peso corporal medio final. S: supervivencia. NTJ: número total de juveniles vivos $\mathrm{L}^{-1}$.

El desempeño productivo hasta juveniles no presentó diferencia en la longitud total, no obstante, el peso final fue disminuyendo con diferencias significativas $(p<0,05)$ entre tratamientos (T1 vs $T 2$, T3 vs $T 4, T 5$ y T6) a medida que aumentaba la densidad poblacional, tal como lo reportado por Liu et al. (2018) para juveniles de Oreochromis niloticus cultivados en biofloc y contrario a lo encontrado para el cultivo en biofloc de juveniles Clarias gariepinus por Fauji et al. (2018) y Corydoras aeneus (Diatin et al., 2019).

La supervivencia de los juveniles de P. orinoquensis cultivados en biofloc fue mayor cuando las densidades poblacionales fueron menores, siendo una relación inversa que presentó diferencias significativas $(p<0,05)$ entre tratamientos (T1 vs T2 y T3 y T4 Vs T5 y T6) similar a lo reportado para el alevinaje de especies como Oreochromis niloticus, Corydoras aeneus, Clarias gariepinus, cultivadas en biofloc a diferentes densidades de siembra (Liu et al., 2018; Fauji et al., 2018; Bittencourt et al., 2019; Diatin et al., 2019). No obstante, cuando se analiza el número total de juveniles vivos al final del experimento (NTJ), se invierte la diferencia entre tratamientos, y la relación entre densidad y número de juveniles es directa, siendo mayor el NTJ a mayor densidad poblacional. 

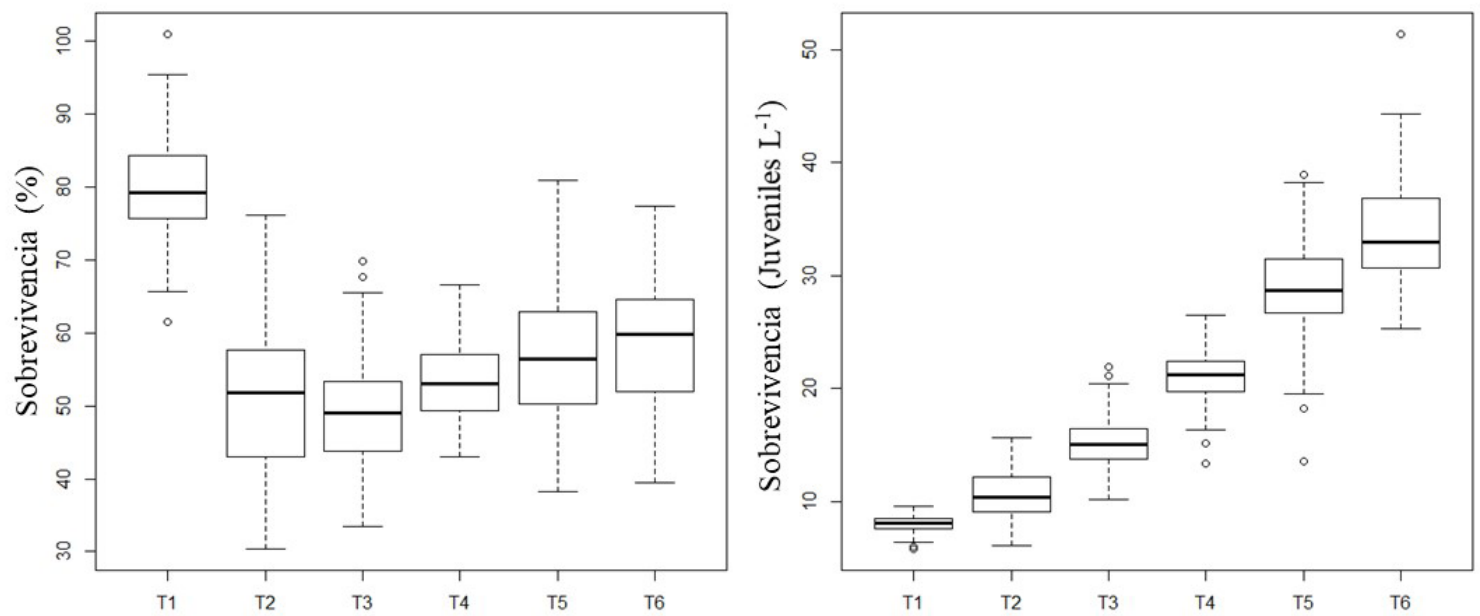

Figura 2. Sobrevivencia en porcentaje y número de juveniles $L^{-1}$ de $P$. orinoquensis después de 12 días de cultivo (del día 13 al 24 DPS) con tecnología biofloc a diferentes densidades de siembra (Kruskal-Wallis test with a p.value: 0,05)

El análisis canónico multivariado al final del experimento arroja supervivencias significativamente diferentes $(p<0,05)$ entre los tratamientos T1, T2 y T3 y estos a su vez con los tratamientos T4, T5 y T6, tal como se muestra en el cuadro 5, siendo el tratamiento con las mejores respuestas el T3, similar a lo encontrado por Poli et al. (2015) para Rhamdia quelen, trabajo en el que se obtuvo una supervivencia entre 38,1-54,4 \% a densidad de 25 larvas L-1; y mayor a lo reportado para juveniles de tilapia (Oreochromis niloticus), donde Ekasari et al. (2015) reportaron 10 larvas $\mathrm{L}^{-1}$, coincidiendo con la densidad reportada por Diatin et al. (2019) para Corydoras aeneus. En el caso de Clarias gariepinus, Fauji et al. (2018) reportaron condiciones similares durante el alevinaje en biofloc a las encontradas en este trabajo, concluyendo que, a un mayor número de peces producidos, estos presentan un menor peso.

\section{CONCLUSIÓN}

La posibilidad de cultivar larvas y juveniles de Piaractus orinoquensis de manera superintensiva con tecnología biofloc es factible en las condiciones reportadas a una densidad de 30 larvas $\mathrm{L}^{-1}$, siendo una alternativa para la producción de semilla de la especie.

\section{REFERENCIAS}

ANDRADE-VERA, SOLANGE; BONIFAZ, MARIA-JOSÉ; DOMÍNGUEZ-GRANDA, L.; MARÍN-JARRÍN, JOSÉ. Uso de la zona de rompiente de playas arenosas por larvas de corvina (Cynoscion spp.) Provincia del Guayas. Ecuador. Latin american journal of aquatic research, v. 45, n. 2, 2017, p. 431-442.

AMERICAN PUBLIC HEALTH ASSOCIATION (APHA). Standard Methods for the examination of Wáter and Wastewater. Washington DC (USA): 2017, part 2540, p, 2-6.

ATENCIO-GARCIA, VICTOR. Producción de juveniles de especies nativas. Revista MVZ Córdoba, v. 6, n. 1, 2001, p. 9-14. https://doi.org/10.21897/rmvz.1060

AVNIMELECH, YORAM. Biofloc technology - A practical guide book. 3rd ed. Baton Rouge (United States): The World Aquaculture Society, 2015, p. 38-65, ISBN: 978-188880-7226

AVNIMELECH, YORAM. Feeding with microbial flocs by tilapia in minimal discharge bioflocs technology ponds. Aquaculture, v. 264, n. 1-4, 2007, p. 140-147. https://doi.org/10.1016/j.aquaculture.2006.11.025 
AYAZO-GENES, J.; PERTUZ-BUELVAS, V.; JIMÉNEZ-VELÁSQUEZ, C.; ESPINOSA-ARAUJO, J.; ATENCIO-GARCÍA, V.; PRIETO-GUEVARA, M. Comunidades planctónicas y bacterianas asociadas al cultivo de bocachico Prochilodus magdalenae con tecnología biofloc. Revista MVZ Córdoba, v. 24, n. 2, 2019, p. 7209-7217. https://doi.org/10.21897/rmvz.1648

AZIM, M.E.; LITTLE, D.C.; BRON, J.E. Microbial protein production in activated suspension tanks manipulating C:N ratio in feed and the implications for fish culture. Bioresource Technology, v. 99, n. 9, 2008, p. 3590-3599. https://doi.org/10.1016/j.biortech.2007.07.063

BAKAR, N.S.A.; NASIR, N.M.; LANANAN, F.; HAMID, S.H.A.; LAM, S.S.; JUSOH A. Optimization of C/N ratios for nutrient removal in aquaculture system culturing African catfish, (Clarias gariepinus) utilizing Bioflocs Technology. International Biodeterioration \& Biodegradation, v. 102, 2015, p. 100-106. http://dx.doi.org/10.1016/j.ibiod.2015.04.001

BALÓN, EUGENE K. Reflections on Some Decisive Events in the Early Life of Fishes. Transactions of the American Fisheries Society, v. 113, n. 2, 1984, p. 178-185. http://dx.doi.org/10.1577/1548-8659(1984)113<178:ROSDEI>2.0.CO2

BARBIERI, EDISON; VIGLIAR-BONDIOLI, ANA-CRISTINA. Acute toxicity of ammonia in Pacu fish (Piaractus mesopotamicus, Holmberg, 1887) at different temperatures levels. Aquaculture Research, v. 46, n. 3, 2013, p. 565-571. https://doi.org/10.1111/are.12203

BALDISSEROTTO, BERNARDO. Fisiologia de Peixes Aplicada a Piscicultura. 3 ed. Santa María (Brazil): editora UFSM, 2013, p. 139-156, ISBN 978.85-7391-198-5.

BITTENCOURT-VIEIRA, RAFAEL; MELO-BARRETO, LEOPOLDO; ZANOTI-FONSECA, KARINA; SANTANALORDELO, MAURICIO; RIBEIRO DE SOUZA, FABIANA; EVANGELISTA-BARRETO, NORMA-SUELY. Zootechnical performance evaluation of the use of biofloc technology in nile tilapia fingerling production at different densities. Boletim do Instituto de Pesca, v. 45, n. 4 e505, 2019, p. 4-7. http://DOI:10.20950/1678-2305.2019.45.4.505

BOURNE, R. Fundamentals of Digital Imaging in Medicine. Springer. New York (USA): 2010,185 p, ISBN 978-184882-086-9.

BROWDY, CRAI L.; RAY, ANDREW J.; LEFFLER, JHON W.; AVIMELECH, YORAM. Biofloc-based aquaculture system. En TIDWELL, JAMES-H; Acuacultura Production Systems. Wiley-Blackwell is an imprint of John Wiley \& Sons, formed by the merger of Wiley's global Scientific, Technical, and Medical business with Blackwell Publishing, 2012, 290 p.

CRAB, ROSELIEN; AVNIMELECH, YORAM; DEFOIRDT,TOM; BOSSIER, PETER; VERSTRAETE, WILLY. Nitrogen removal techniques in aquaculture for a sustainable production. Aquaculture, v. 270, n. 1-4, 2007, p. 1-14. https://doi.org/10.1016/j.aquaculture.2007.05.006

DAVID-RUALES, CARLOS-DAVID; MACHADO-FRACALOSSI, DEVORA; VÁSQUEZ-TORRES, WALTER. Desarrollo temprano en larvas de peces. clave para el inicio de la alimentación exógena. Revista Lasallista de Investigación, v.15, n. 1, 2018, p. 180 - 194.

https://doi.org/10.22507/rli.v15n1a10

DE SCHRYVER, P.; CRAB, R.; DEFOIRDT, T.; BOON, N.; VERSTRAETE, W. The basics of bio-flocs technology: The added value for aquaculture. Aquaculture, v. 277, n. 3-4, 2008, p 125-137.

https://doi.org/10.1016/j.aquaculture.2008.02.019

DIATIN, ES; MUHAMMAD, AGUS-SUPRAYUDI; BUDIARDI, TATAG; HARRIS. ENANG; WIDANARNI, WIDANANRNI. Biofloc technology on the intensive aquaculture of bronze corydoras ornamental fish Corydoras aeneus with different stocking densities. Journal Akuakultur Indonesia, v. 18, n. 2, 2019, p. 202-213. https://doi.org/10.19027/jai.18.2.202-213

EBELING, JAMES; TIMMONS, MICHAEL B.; BISOGNI, J.J. Engineering analysis of the stoichiometry of photoautotrophic. autotrophic. and heterotrophic removal of ammonia-nitrogen in aquaculture systems. Aquaculture, v. 257, n. 1-4, 2006, p. 346-358.

https://doi.org/10.1016/j.aquaculture.2006.03.019 
EKASARI, JULIE; RIVANDI, DIO-RHEZA; FIRDAUSI, AMALIA-PUTRI; SURAWIDJAJA, ENANG-HARRIS; ZAIRIN, MUHAMMAD; BOSSIER, PETER; DE SCHRYVER, PETER. Biofloc technology positively affects Nile tilapia (Oreochromis niloticus) larvae performance. Aquaculture, v.441, 2015, p. 72-77. https://doi.org/10.1016/j.aquaculture.2015.02.019

EKASARI, JULIE; SUPRAYUDI, MUHAMMAD-AUGUS; WIYOTO, WIYOTO; HAZANAH, RATIH-FAUZIATIN; LENGGARA, GILANG-SATYA; SULISTIANI, ROSI; ALKAHFI, MUHAMMAD; ZAIRIN JR, MUHAMMAD. Biofloc technology application in African catfish fingerling production: The effects on the reproductive performance of broodstock and the quality of eggs and larvae. Aquaculture, v. 464, 2016, p.349-356. https://doi.org/10.1016/j.aquaculture.2016.07.013

EMERENCIANO, MAURICIO; CÓRDOVA-MARTINEZ, LUIS-RAFAEL; MARTÍNEZ-PORCHAS, MARCEL; MIRANDA-BAEZA, ANSELMO. Biofloc Technology (BFT): A Tool for Water Quality Management in Aquaculture en Water Quality Edited by Hlanganani Tutu. ISBN 978-953-51-2881-6. pp 426. Publisher: InTech. Chapters published January 18. 2017 under CC BY 3.0 license. http://DOI:10.5772/66416

EMERSON, KENNETH; RUSSO, ROSEMARIE C.; LUND, RICHARD E.; THURSTON, ROBERT V. Aqueous ammonia equilibrium calculations: effect of $\mathrm{pH}$ and temperature. Journal of the Fisheries Board of Canada, v. 32, n. 12,1975 , p. 2379-2383. https://doi.org/10.1139/f75-274

FAUJI, HILMI; BUDIARDI, TATAG; EKASARI, JULIE. Growth performance and robustness of African Catfish Clarias gariepinus (Burchell) in biofloc-based nursery production with different stocking densities. Aquaculture Research, v. 49, n. 3, 2018, p. 1339-1346. https://doi.org/10.1111/are.13595

GARCÍA-RÍOS, LOMBARDO; MIRANDA-BAEZA, ANSELMO; COELHO-EMERENCIANO, MAURICIOGUSTAVO; HUERTA-RÁBAGO, JOSÉ-ALBERTO; OSUNA-AMARILLAS, PABLO. Biofloc technology (BFT) applied to tilapia fingerlings production using different carbon sources: Emphasis on commercial applications. Aquaculture, v. 502, 2019, p. 26-31. https://doi.org/10.1016/j.aquaculture.2018.11.057

GOMES-VILANI, FELIPE; SCHVEITZER, RODRIGO; DA FONSECA-ARANTES, RAFAEL; DO NASCIMENTOVIEIRA, FELIPE; MANOEL- DO ESPÍRITO SANTO, CARLOS; QUADROS-SEIFFERT, WALTER. Strategies for water preparation in a biofloc system: Effects of carbon source and fertilization dose on water quality and shrimp performance. Aquacultural Engineering, v. 74, 2016, p. 70-75. https://doi.org/10.1016/j.aquaeng.2016.06.002

HARGREAVES, JHON A. Biofloc production systems for aquaculture. Southern Regional Aquaculture Center SRAC Publication, n. 4503, 2013, 12 p.

HERNÁNDEZ-ESTRADA, ROBERTO; RODRÍGUEZ-MARTINEZ, ANDREA; RUÍZ_MARTINEZ, OSIRIS; MONROY-DOSTA, MARIA-DEL CARMEN. Ecological succession of plankton in a biofloc system with molasses as carbon source. Scientific Journal of Biological Science, v, 6, n. 7, 2017, p. 222-228. https://doi:10.14196/sjbs.v6i7.2456

JIMÉNEZ-OJEDA, YIRA-KARINA; COLLAZOS-LASSO, LUIS-FELIPE; ARIAS-CASTELLANOS, JOSÉ-ALFREDO. Dynamics and use of nitrogen in Biofloc Technology - BFT. AACL Bioflux, v. 11, n. 4, 2018, p 1107-1129.

KARASU-BENLI, A.; KOKSAL, G. The acute toxicity of Ammonia on Tilapia (Oreochromis niloticus) Larvae and fingerling. Turkish Journal of Veterinary and Animal Sciences, v. 29, 2005, p 339-344.

KUBITZA, F. Criação de tilapias em sistema com bioflocos sem renovação de agua. Panorama da aqüicultura, v. 21, n 125, 2011, p 14-23.

KUBITZA, F. A relação entre pH. gás carbônico. alcalinidade e dureza sua influência no desempenho e saúde dos peixes e camarões. Rev. Panorama de aqüicultura. 2017. https://panoramadaaquicultura.com.br/a-aguana-aquicultura-parte-2/ [consultado febrero 10 de 2021].

LI, JIAWEI; LIU, GANG; LI, CHANGWEI; DENG, YALE; TADDA, MUSA-ABUBAKAR; LAN, LIHUA; ZHU, SONGMING; LIU, DEZHAO. Effects of different solid carbon sources on water quality, biofloc quality and gut microbiota of Nile tilapia (Oreochromis niloticus) larvae. Aquaculture, v. 495, 2018, p. 919-931. https://doi.org/10.1016/j.aquaculture.2018.06.078 
LIU, GANG; YE, ZHANGYING; LIU, DEZHAO; ZHAO, JIAN; SIVARAMASAMY, ELAYARAJA; DENG, YALRE; ZHU, SONGMING. Influence of stocking density on growth, digestive enzyme activities, immune responses, antioxidant of Oreochromis niloticus fingerlings in biofloc systems. Fish and Shellfish Immunology, v. 81, 2018, p. 416-422. https://doi.org/10.1016/j.fsi.2018.07.047

LUO, GUOZHI; ZHANG, NAN; CAI, SHENGLI; TAN, HONGXIN; LIU, ZEFENG. Nitrogen dynamics, bacterial community composition and biofloc quality in biofloc-based systems cultured Oreochromis niloticus with poly$\beta$-hydroxybutyric and polycaprolactone as external carbohydrates. Aquaculture, v. 479, 2017, p. 732-741. https://doi.org/10.1016/j.aquaculture.2017.07.017

MAYA-GUTIÉRREZ, STHEPHANIEE; MONROY-DOSTA, MARÍA DEL CARMEN; HANDAM-PARTIDA, AIDA; CASTRO-MEJÍA, JORGE; RODRÍGUEZ-MONTES DE OCA, GUSTAVO-ALEJANDRO. Effect of two carbon sources in microbial abundance in a Biofloc culture system with Oreochromis niloticus (Linnaeus. 1758). International Journal of Fisheries and Aquatic Studies, v. 4, n. 3, 2016, p. 421-427.

MARTINS, G.B.; TAROUCO, F.; ROSA, C.E.; ROBALDO, R.B. The utilization of sodium bicarbonate, calcium carbonate or hydroxide in biofloc system: water quality, growth performance and oxidative stress of Nile tilapia (O.niloticus). Aquaculture, v. 468, n. 1, 2017, p.10-17.

https://doi.org/10.1016/j.aquaculture.2016.09.046

PÉREZ-FUENTES, JORGE A.; HERNANDEZ-VERGARA, MARTHA; PEREZ-ROSTRO, CARLOS; FOGEL, IRA C. $\mathrm{N}$ ratios affect nitrogen removal and production of Nile tilapia Oreochromis niloticus raised in a biofloc system under high density cultivation. Aquaculture, v. 452, 2016, p. 247-251. https://doi.org/10.1016/j.aquaculture.2015.11.010

POLI, MOISES-ANGEL; SCHVEITZER, RODRIGO; DE OLIVEIRA NUÑER, ALEX. The use of biofloc technology in a South American catfish (Rhamdia quelen) hatchery: Effect of suspended solids in the performance of larvae. Aquacultural Engineering, v. 66, 2015, p. 17-21. https://doi.org/10.1016/j.aquaeng.2015.01.004

PRIETO, MARTHA; ATENCIO, VICTOR. Zooplankton in larviculture of neotropical fishes. Revista MVZ Córdoba, v. 13, n. 2, 2008, p. 1416-1417. https://doi.org/10.21897/rmvz.401

RAY, A.J.; SEABORN, G.; LEFFLER, J.W.; WILDE, S.B.; LAWSON, A.; Y BROWDY, C.L. Characterization of microbial communities in minimal-exchange, intensive aquaculture systems and the effects of suspended solids management. Aquaculture, v. 310, n. 1-2, 2010, p. 130-138. https://doi.org/10.1016/j.aquaculture.2010.10.019

SISTEMA DE INFORMACIÓN DE GESTIÓN Y DESEMPEÑO DE ORGANIZACIÓN DE CADENAS (SIOC), MINISTERIO DE AGRICULTURA Y DESARROLLO RURAL DE COLOMBIA. Producción de la Acuicultura 2010 2019. 2020. https://sioc.minagricultura.gov.co/Acuicultura/Documentos/2019-12-31\%20 Cifras\%20Sectoriales.pdf [consultado marzo 7 de 2021].

XU, W.J.; MORRIS, T.C.; SAMOCHA, T.M. Effects of $\mathrm{C} / \mathrm{N}$ ratio on biofloc development, water quality, and performance of Litopenaeus vannamei juveniles in a biofloc-based, high-density, zero-exchange, outdoor tank system. Aquaculture, v. 453, 2016, p. 169-175. https://doi.org/10.1016/j.aquaculture.2015.11.021

ZAPATA-LOVERA, KATHERINE-PATRICIA; BRITO, LUIS-OTAVIO; DE LIMA, PRISCILLA-CELES-MACIEL; VINATEA-ARANA, LUIS-ALEJANDRO; GALVEZ, ALFREDO-OLIVERA; CÁRDENAS-VARGAS, JESSIEMARINA. Cultivo de alevines de tilapia en sistema biofloc bajo diferentes relaciones carbono/nitrógeno. Boletim do Instituto de Pesca. São Paulo, v. 43, n. 3, 2017, p. 399 - 407.

https://doi.org/10.20950/1678-2305.2017v43n3p399 